\title{
The VP16 transcription activation domain is functional when targeted to a promoter-proximal RNA sequence
}

\author{
Laurence S. Tiley, ${ }^{1,4}$ Steven J. Madore, ${ }^{1}$ Michael H. Malim, ${ }^{2,5}$ and Bryan R. Cullen ${ }^{1,3}$ \\ ${ }^{1}$ Howard Hughes Medical Institute, ${ }^{3}$ Section of Genetics, and Departments of ${ }^{2}$ Medicine and ${ }^{3}$ Microbiology, Duke \\ University Medical Center, Durham, North Carolina 27710 USA
}

\begin{abstract}
Among eukaryotic transcription trans-activators, the human immunodeficiency virus type 1 (HIV-1) Tat protein is exceptional in that its target site TAR is an RNA rather than a DNA sequence. Here, we confirm that fusion of Tat to the RNA-binding domain of the HIV-1 Rev protein permits the efficient activation of an HIV-1 long terminal repeat (LTR) promoter in which critical TAR sequences have been replaced by RNA sequences derived from the HIV-1 Rev response element (RRE). An RRE target sequence as small as 13 nucleotides is shown to form an effective in vivo target for Rev binding. More important, a fusion protein consisting of Rev attached to the VP16 transcription activation domain was also observed to efficiently activate the HIV-1 LTR from this nascent RNA target. These data demonstrate that trans-activation of transcription by acidic activation domains does not require a stable interaction with the promoter DNA and suggest that VP16, like Tat, can act on steps subsequent to the formation of the HIV-1 LTR preinitiation complex. The finding that the activation domains of VP16 and Tat are functionally interchangeable raises the possibility that these apparently disparate viral trans-activators may nevertheless act via similar mechanisms.
\end{abstract}

[Key Words: Trans-activator; HIV-1; acidic activation domains; Rev; Tat]

Received July 14, 1992; revised version accepted August 26, 1992.

Replication of the human immunodeficiency virus type 1 (HIV-1) is critically dependent on the functional expression of the virally encoded nuclear regulatory proteins Tat and Rev (Dayton et al. 1986; Fisher et al. 1986; Sodroski et al. 1986). Tat serves to trans-activate the expression of genes that are under the control of the HIV-1 long terminal repeat (LTR) promoter element and can therefore be viewed as functionally comparable to the transcriptional trans-activators encoded by other complex retroviruses or DNA tumor viruses. Tat, however, is unique in that its target site is not a DNA sequence but, rather, a 59-nucleotide RNA stem-loop structure, termed TAR, located immediately $3^{\prime}$ to the viral mRNA cap site (Feng and Holland 1988; Hauber and Cullen 1988; Jakobovits et al. 1988; Berkhout and Jeang 1989). The direct interaction of Tat with this nascent RNA sequence (Berkhout et al. 1989; Dingwall et al. 1990; Roy et al. 1990; Weeks et al. 1990) has been shown to result in a marked increase in the rate of transcription from the HIV-1 LTR (Hauber et al. 1987; Kao et al. 1987; Jakobovits et al. 1988). It has been proposed that Tat acts primarily by enhancing the elongation effi-

Present addresses: ${ }^{4}$ Department of Virology, Bristol Myers Squibb Pharmaceutical Research Institute, Princeton, New Jersey 08543 USA; ${ }^{5}$ Howard Hughes Medical Institute and Department of Microbiology, University of Pennsylvania, Philadelphia, Pennsylvania 19104 USA. ciency of otherwise poorly processive RNA polymerase II molecules that have initiated within the HIV-1 LTR promoter (Kao et al. 1987; Marciniak et al. 1990; Feinberg et al. 1991; Kato et al. 1992). In contrast, conventional eukaryotic transcriptional trans-activators interact with DNA target sites and are believed to act primarily at the level of initiation (for review, see Roeder 1991).

The HIV-1 Rev protein acts post-transcriptionally to induce the cytoplasmic expression of unspliced and singly spliced mRNA species that encode the viral structural proteins (Emerman et al. 1989; Hammarskjold et al. 1989; Malim et al. 1989b). These viral transcripts bear a single copy of the cis-acting RNA target sequence for Rev, the 234-nucleotide Rev response element (RRE) (Rosen et al. 1988; Malim et al. 1989b). Rev, like Tat, has been shown to bind its RNA target site with high affinity in vitro (Daly et al. 1989; Zapp and Green 1989; Malim et al. 1990). Previously, it was reported that a Tat-Rev (Tat/Rev) fusion protein could activate transcription from an HIV-1 promoter construct in which the TAR element had been replaced with the entire 234-nucleotide RRE (Southgate et al. 1990). We have used a more refined version of this approach to define amino acid and nucleotide sequence requirements for the in vivo interaction of Rev with the RRE. More importantly, we demonstrate that a fusion protein consisting of Rev attached to the acidic transcriptional activation domain of the 
herpes simplex virus (HSV)-derived VP16 trans-activator (Sadowski et al. 1988; Cress and Triezenberg 1991) can efficiently activate transcription from the HIV-1 LTR when targeted to an RRE-derived RNA target. These results demonstrate that a representative DNA sequencespecific eukaryotic trans-activator can also function when targeted to a promoter proximal RNA target sequence and raise the possibility that despite its unique RNA target specificity, Tat may well activate transcription by a conventional mechanism.

\section{Results}

TAR, the cis-acting RNA target of the HIV-1 Tat transactivator, is a 59-nucleotide RNA stem-loop located at the 5' end of all HIV-1 transcripts (Hauber and Cullen 1988; Jakobovits et al. 1988). Maintenance of the helical structure of TAR is critical for the appropriate presentation of essential primary RNA sequence information located in the terminal hexanucleotide loop and an adjacent pyrimidine-rich bulge (Fig. 1) (Feng and Holland 1988; Berkhout and Jeang 1989; Roy et al. 1990). These sequences are believed to act as in vivo-binding sites for Tat as well as for at least one essential cellular cofactor (Dingwall et al. 1990; Roy et al. 1990; Weeks et al. 1990; for review, see Cullen 1990).

The stem-loop IIB (SLIIB) subdomain of the HIV-1 RRE is necessary and sufficent for Rev binding in vitro and is critical for RRE function in vivo (Malim et al. 1990; Bartel et al. 1991; Heaphy et al. 1991; Tiley et al. 1992; Kjems et al. 1992). To test whether the SLIIB sequence is also sufficient for Rev binding in vivo, we substituted a 29-nucleotide SLIIB RNA sequence in place of the essential apical region of TAR (Fig. 1). We then compared the ability of Tat, Rev, and a Tat/Rev fusion protein to activate expression from constructs in which either the wild-type HIV-1 LTR (pTAR/CAT) or an LTR containing the SLIIB substitution in TAR (pSLIIB/CAT) was fused to the chloramphenicol acetyl transferase (cat) indicator gene. In this transient expression assay in HeLa cells, Tat and Tat/Rev, but not Rev, were found to efficiently activate HIV-1 LTR gene expression via the TAR element (Table 1). Neither Tat nor Rev, however, was active on the construct in which the TAR element had been rendered nonfunctional by replacement of critical TAR nucleotides with the RRE SLIIB sequence. In contrast, the Tat/Rev fusion protein activated expression from the pSLIIB/CAT construct by $60-$ to $>100$-fold.

We have described previously (Malim et al. 1989a,b) an assay for Rev function in transfected COS cells based on the ability of Rev to induce the expression of a truncated, single-exon form of the viral Tat protein from the genomic Tat expression construct pgTat. This short form of the Tat protein is encoded by an unspliced viral mRNA whose nucleocytoplasmic export is dependent on Rev (Malim et al. 1989b). As shown in Figure 2, the singleexon form of Tat is induced on cotransfection of pgTat with either Rev (Fig. 2, lane 3) or the Tat/Rev fusion protein (Fig. 2, lane 4), although the latter clearly displays reduced Rev activity. This immunoprecipitation

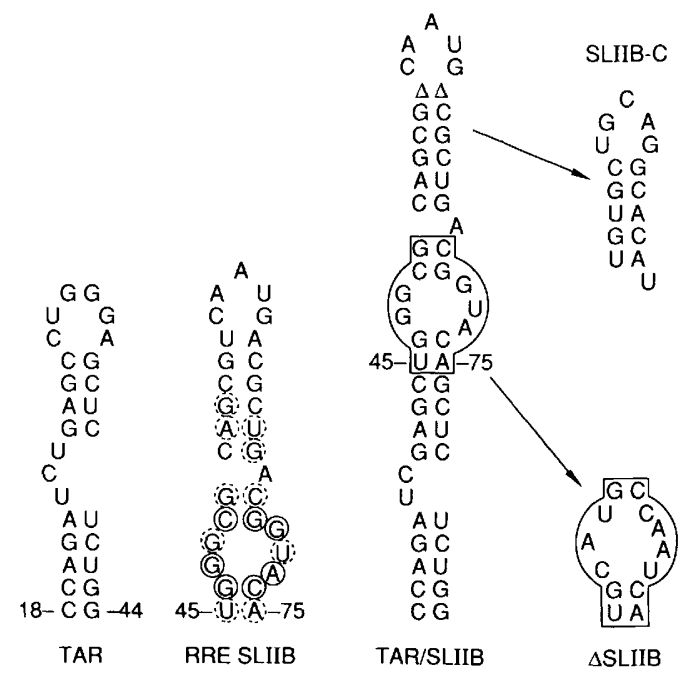

Figure 1. Predicted structure of wild-type and synthetic RNA target sequences. (TAR) The sequence and predicted structure of the minimal functional TAR element, which extends from +18 to +44 within the full-length 59-nucleotide TAR structure (Jakobovits et al. 1988). (RRE SLIIB) The minimal RNA sequence shown to be both necessary and sufficient for Rev binding in vitro. This SLIIB sequence extends from nucleotide 45 to nucleotide 75 within the 234-nucleotide RRE. Individual nucleotides that have been proposed to make major or minor contributions to sequence-specific recognition of the RRE by Rev are indicated by closed or broken circles, respectively. (TAR/SLIIB) In the pSLIIB/CAT construct, the apical region of TAR, including the essential hexanucleotide loop, was replaced by the SLIIB sequence. Triangles indicate two deleted nucleotides relative to the wild-type sequence. The boxed SLIIB sequence contains all nucleotides mapped as critical to SLIIB recognition in vitro and has been proposed to form the core Rev recognition site (Heaphy et al. 1991). The complete predicted TAR/SLIIB structure is 81-nucleotides in length. (SLIIB-C) The 16-nucleotide apical region of SLIIB was replaced with the indicated 16-nucleotide sequence in the pSLIIB-C/CAT plasmid. This substitution mutation is designed to maintain the structure of this helical RNA element. ( $\triangle$ SLIIB) The proposed 13-nucleotide core Rev recognition site of SLIIB was substituted with the indicated sequence to give $\mathrm{p} \Delta \mathrm{SLIIB} / \mathrm{CAT}$. This substitution mutation alters 7 of the 13 nucleotides in this core sequence without markedly affecting the predicted RNA structure of the larger TAR/SLIIB sequence.

assay in COS cells also facilitated analysis of the level of expression and relative mobility of the various Tat and Rev fusion proteins described below.

To assess whether the activation of the HIV-1 LTR promoter by the Tat/Rev fusion protein reflected the targeting of the Tat activation domain to the SLIIB sequence by the Rev RNA-binding motif, we introduced mutations that have been shown previously (Malim and Cullen 1991) to inactivate either the arginine-rich RNAbinding domain (Tat/M5) or the leucine-rich activation domain (Tat/M10) of Rev. As predicted, these mutations also inactivated Rev function in the context of the Tat/ Rev fusion protein (Fig. 2, lanes 4-6). Whereas both fusion proteins fully retained the ability to activate the HIV-1 LTR via the TAR element, only the Tat/M10 con- 
Table 1. Trans-activation of the HIV-1 LTR by an RRE-derived RNA target

\begin{tabular}{cccc}
\hline & \multicolumn{3}{c}{ Relative trans-activation } \\
\cline { 2 - 4 } Clones transfected & exp. 1 & exp. 2 & exp. 3 \\
\hline pTAR/CAT + pcTat & 130 & 114 & 152 \\
+ pcRev & $<1$ & $<1$ & - \\
+ pcTat/Rev & 41 & 62 & 82 \\
+ pcTat/M5 & 119 & 68 & 88 \\
+ pcTat/M10 & - & 69 & 93 \\
+ pK41A/Rev & $<1$ & $<1$ & - \\
+ pcRev & 1.8 & $<1$ & 1.4 \\
+ pcTat/Rev & 89 & $<1$ & - \\
+ pcTat/M5 & 2.6 & 61 & 60 \\
+ pcTat/M10 & - & 35 & 3.4 \\
+ pK41A/Rev & $<1$ & $<1$ & - \\
pSLIIB/CAT & $<1.3$ \\
\hline
\end{tabular}

HeLa cell cultures $(35 \mathrm{~mm})$ were transfected with equal amounts $(2 \mu \mathrm{g})$ of the indicated expression plasmids using the $\mathrm{CaPO}_{4}$ procedure (Cullen 1987). At $48 \mathrm{hr}$ after transfection, cells were harvested and extracted, and the level of CAT enzyme activity was determined by the diffusion method of Neumann et al. (1987). Relative trans-activation is presented as a multiple of the basal activity of each construct as determined by cotransfection with the parental $\mathrm{pBC} 12 / \mathrm{CMV}$ expression plasmid. Values were adjusted for minor variability in the level of total protein in each extract, as determined by the method of Bradford (1976). Three representative experiments are shown.

struct retained significant activity on the SLIIB/CAT indicator plasmid (Table 1). We therefore conclude that trans-activation by Tat/Rev is dependent on an intact Rev RNA-binding domain but does not require Rev function per se.

Mutational analysis of Tat has defined a short amino acid sequence, termed the core element, that is critical for Tat function in vivo (Green et al. 1989; Tiley et al. 1990; Carroll et al. 1991). Fusion of rev to a tat gene bearing a point mutation within this sequence (Lys$41 \rightarrow$ Ala) resulted in a chimeric protein $(\mathrm{K} 41 \mathrm{~A} / \mathrm{Rev})$ that retained full Rev activity (data not shown) but was inactive on both the TAR element and on the SLIIB RNA target (Table 1). We therefore conclude that the functional interaction of Tat/Rev with SLIIB requires an intact Tat activation sequence.

\section{RNA sequence specificity of Rev in vivo}

The in vitro RNA sequence specificity of Rev has been analyzed extensively by mutagenesis, modification interference analysis, and in vitro selection (Bartel et al. 1991; Heaphy et al. 1991; Kjems et al. 1992; Tiley et al. 1992). These reports have established that the overall structure of the SLIIB RNA is critical for Rev binding and have also identified several nucleotide residues that are critical for, or that contribute toward, RNA binding by Rev (Fig. 1). In general, these critical nucleotide residues were found to cluster within a small, 13-nucleotide core that is predicted to form a stem-bulge-stem structure.
The adjacent helical part of SLIIB serves an essential structural role and may also contribute to the sequence specificity of the Rev-RRE interaction (Fig. 1). To test whether these in vitro observations are relevant to RNA binding by Rev in vivo, we constructed two derivatives of the pSLIIB/CAT indicator construct. In the p $\Delta$ SLIIB/ CAT plasmid, we mutated 7 of 13 nucleotides within the core Rev-binding site (Fig. 1). In contrast, in the pSLIIB$\mathrm{C} / \mathrm{CAT}$ plasmid, this 13-nucleotide sequence was left undisturbed, whereas the 16-nucleotide apical region of SLIIB was replaced with an unrelated sequence that was predicted to maintain a comparable secondary structure (Fig. 1).

Both the $\mathrm{p} \Delta$ SLIIB/CAT and the pSLIIB-C/CAT plas-

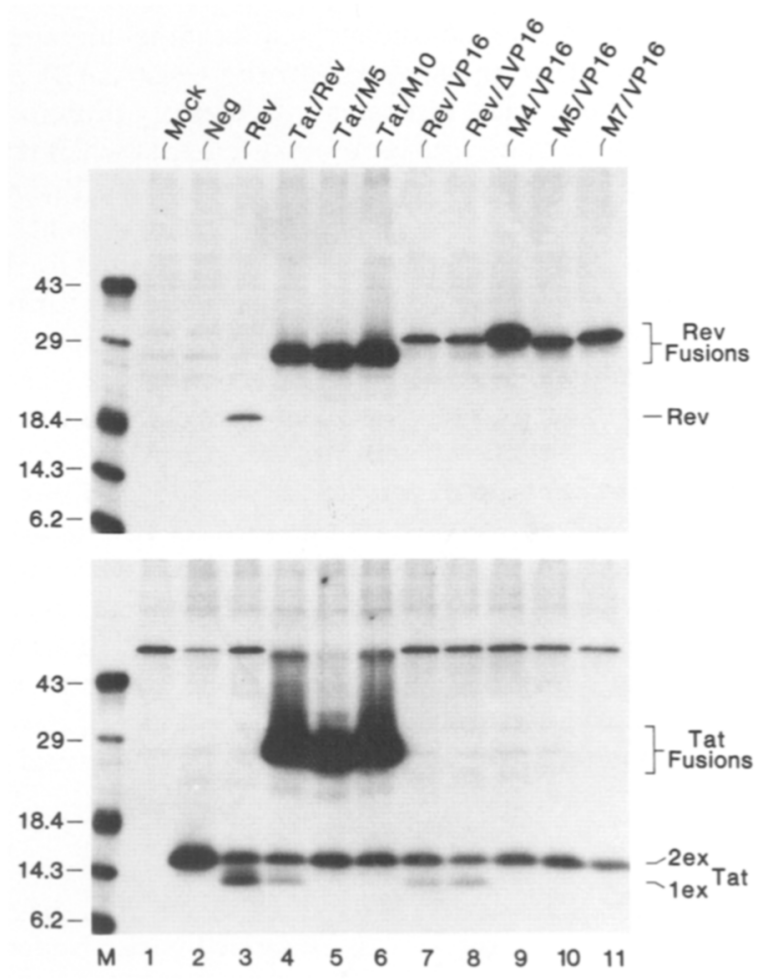

Figure 2. Immunoprecipitation of HIV-1 Rev and Tat proteins. COS cell cultures were cotransfected with the indicator construct pgTat and the negative control plasmid $\mathrm{pBC} 12 / \mathrm{CMV}$ (lane 2), the wild-type Rev expression vector pcRev (lane 3), or plasmids expressing the indicated wild-type or mutant Rev fusion protein. At $48 \mathrm{hr}$ after transfection, cultures were labeled with $\left[{ }^{35} \mathrm{~S}\right]$ cysteine and lysed in RIPA buffer. Equal aliquots of each cleared lysate were subjected to immunoprecipitation using rabbit antisera specific for either Rev (top) or for Tat (bottom $\mid$. Wild-type Rev migrates at $\sim 19 \mathrm{kD}$, whereas the various Rev fusion proteins migrate at $\sim 27 \mathrm{kDa}$. In the absence of Rev, the pgTat indicator construct expresses exclusively a fully spliced mRNA that encodes the $\sim 16-\mathrm{kD} 2$ exon (2ex) form of Tat (lane 2). In the presence of Rev, cytoplasmic expression of an incompletely spliced mRNA that encodes the $\sim 14-\mathrm{kD}$ single exon (1ex) form of Tat is induced (lane 3). Appearance of this truncated Tat protein is therefore diagnostic of Rev function (Malim et al. 1989a). (Mock) Mock-transfected culture; (M) radioactive protein molecular mass markers. 
mids were, as expected, refractory to transactivation by Tat (Table 2). The response of the $\mathrm{p} \Delta$ SLIIB/CAT construct to the Tat/Rev fusion protein was inhibited by $\sim 95 \%$ when compared with the wild-type pSLIIB/CAT construct. In contrast, pSLIIB-C/CAT, which retains only the predicted 13-nucleotide core RNA-binding site of Rev, was vigorously transactivated by Tat/Rev, displaying $\geqslant 70 \%$ of the response of the wild-type SLIIB sequence. We therefore conclude that the 13-nucleotide sequence element that was predicted to form the core RNA-binding site of Rev on the basis of in vitro analysis is also both necessary and sufficient for specific Rev binding in vivo.

\section{Targeting of the VP16 activation domain to an RNA sequence}

DNA sequence-specific nucleic acid-binding domainsmost notably that encoded within the yeast GAL4 protein-have been used extensively to identify protein sequences that can activate promoter elements when tethered to adjacent DNA sequences (for review, see Ptashne 1988; Mitchell and Tjian 1989). Among the more active of the transcriptional activation domains defined by this approach is that encoded within the carboxy-terminal region of the HSV-1-derived VP16 trans-activator (Sadowksi et al. 1988). The data presented in Tables 1 and 2 demonstrate that the Rev RNA-binding domain can efficiently target a heterologous protein sequence-in this case, Tat-to a promoter proximal RRE-derived RNA sequence. We therefore asked whether the VP16 activation domain (Triezenberg et al. 1988) could exert any effect on the HIV-1 promoter when similarly targeted to the SLIIB sequence. Remarkably, a fusion protein consisting of the carboxy-terminal 78 amino acids of VP16 attached to the carboxyl terminus of Rev was found to activate gene expression directed by the pSLIIB/CAT plasmid by

Table 2. In vivo RNA target specificity of ReV

\begin{tabular}{cccc}
\hline & \multicolumn{3}{c}{ Relative trans-activation } \\
\cline { 2 - 4 } Clones transfected & exp. 1 & exp. 2 & exp. 3 \\
\hline pSLIIB/CAT + pcTat & $<1$ & 1.8 & 1.6 \\
+ pcTat/Rev & 113 & 108 & 116 \\
+ pcRev/VP16 & 31 & 38 & 73 \\
pSLIIB-C/CAT + pcTat & $<1$ & $<1$ & 1.4 \\
+ pcTat/Rev & 103 & 75 & 128 \\
+ pcRev/VP16 & 8.3 & 7.0 & 13.1 \\
pDSLIIB/CAT + pcTat & $<1$ & $<1$ & $<1$ \\
+ pcTat/Rev & 5.5 & 5.1 & 7.2 \\
+ pcRev/VP16 & $<1$ & $<1$ & $<1$ \\
\hline
\end{tabular}

The responsiveness of each RNA target to the indicated transactivators was analyzed in HeLa cells, as described in Table 1. However, each of the cultures assayed here was also cotransfected with a low level $(0.4 \mu \mathrm{g})$ of the internal control plasmid pSV $\beta-$ Gal. Levels of CAT and $\beta$-galactosidase expressed in each culture were assayed in parallel, and the observed CAT activities were then corrected for any minor variations in transfection efficiency revealed by this internal control.
Table 3. The VP16 activation domain can trans-activate the HIV-1 LTR when tethered to an RNA target

\begin{tabular}{cccc}
\hline & \multicolumn{3}{c}{ Relative trans-activation } \\
\cline { 2 - 4 } Clones transfected & exp. 1 & exp. 2 & exp. 3 \\
\hline pSLIIB/CAT + pcRev & 1.1 & $<1$ & 1.1 \\
+ pcTat/Rev & 123 & 169 & 125 \\
+ pcRev/VP16 & 67 & 61 & 39 \\
+ pcRev/AVP16 & 1.5 & 3.1 & 2.8 \\
+ pM4/VP16 & 40 & 37 & 28 \\
+ pM5/VP16 & $<1$ & $<1$ & $<1$ \\
+ pM7/VP16 & 92 & 88 & 69 \\
+ pcRev/VP16 & 125 & 94 & 92 \\
pTAR/CAT + pcTat/Rev & $<1$ & $<1$ & $<1$ \\
\hline
\end{tabular}

Assays were performed in HeLa cells as described in Table 2.

30- to 70 -fold (Table 3). In contrast, the Rev/VP16 protein, which is not predicted to interact with TAR, failed to activate the wild-type HIV-1 LTR. The RNA sequence specificity of this interaction was demonstrated further by the observation that Rev/VP16 displayed a partial activation phenotype when tested on the minimal RRE present in pSLIIB-C/CAT and was inactive when tested on the $\mathrm{p} \Delta$ SLIIB/CAT plasmid (Table 2).

Mutational analysis of the VP16 activation domain has identified Phe-442 as important for in vivo transactivation (Cress and Triezenberg 1991). We therefore tested whether the targeted mutagenesis of this residue (Phe $\rightarrow$ Pro) would affect trans-activation by Rev/VP16. Both the wild-type and mutant (Rev/DVP16) fusion proteins were expressed at comparable levels in transfected cells, and both displayed readily detectable Rev function (Fig. 2, lanes 7,8). However, the ability of the Rev/DVP16 fusion protein to activate gene expression directed by the pSLIIB/CAT indicator construct was inhibited by $>90 \%$ (Table 3). We therefore conclude that VP16 residue 442 is important for transcriptional activation via an RNA target sequence.

Previously, we have described two inactive mutants of Rev, termed M4 and M7, that lack the ability to multimerize in vitro yet retain the ability to bind the RRE as a monomer (Malim and Cullen 1991; Tiley et al. 1992). To examine whether Rev multimerization was important for trans-activation of the HIV-1 LTR by Rev/VP16, we assayed the biological activity of fusion proteins consisting of the VP16 activation domain attached to the carboxyl terminus of either M4 or M7. As a negative control, we also tested a fusion of VP16 with the RNAbinding domain-defective M5 derivative of Rev. Whereas these fusion proteins were expressed at comparable levels in transfected cells, only the parental Rev/VP16 protein displayed detectable Rev activity (Fig. 2, lanes 7-11). As expected, the fusion protein lacking a functional RNA-binding motif (M5/VP16) had also lost the ability to trans-activate the SLIIB/CAT reporter construct ( $\mathrm{Ta}$ ble 3). In contrast, Rev fusion proteins that were predicted to be unable to multimerize, that is, M4/VP16 and M7/VP16, retained the ability to activate CAT ex- 
pression from the pSLIIB/CAT indicator construct ( $\mathrm{Ta}$ ble 3). This observation therefore suggests that activation of the HIV-1 LTR by Rev/VP16 can be mediated by a single RNA-bound VP16 activation domain.

The data presented in Tables 1-3 were derived by transient expression analysis in transfected HeLa cells. We then asked whether these observations could be confirmed in Jurkat, a human $\mathrm{CD} 4^{+} \mathrm{T}$-cell line that is permissive for HIV-1 replication. Analysis of CAT activity in transfected Jurkat cell cultures confirmed that the Tat/Rev fusion protein is as active on the synthetic SLIIB RNA target as on the normal TAR element (Fig. 3). Similarly, Rev/VP16 efficiently trans-activated $(\sim 25$ fold) the HIV-1 LTR present in the pSLIIB/CAT construct but failed to activate the wild-type HIV-1 LTR. The activity of each of these chimeric proteins on the SLIIB target was again totally abrogated by introduction of the M5 mutation into the Rev RNA-binding motif. Comparable results were also obtained by transfection of the monkey cell line COS (data not shown). We therefore conclude that the ability of Tat/Rev and Rev/VP16 to activate the HIV-1 LTR via the introduced SLIIB target is not restricted to HeLa cells.

\section{Both Tat and VP16 require that an RNA target be promoter proximal}

An unusual characteristic of the Tat trans-activator is that transcriptional activation of the HIV-1 LTR is lost rapidly when the TAR element is moved away from the RNA cap site (Selby et al. 1989). To examine the importance of promoter proximity for the function of the TAR

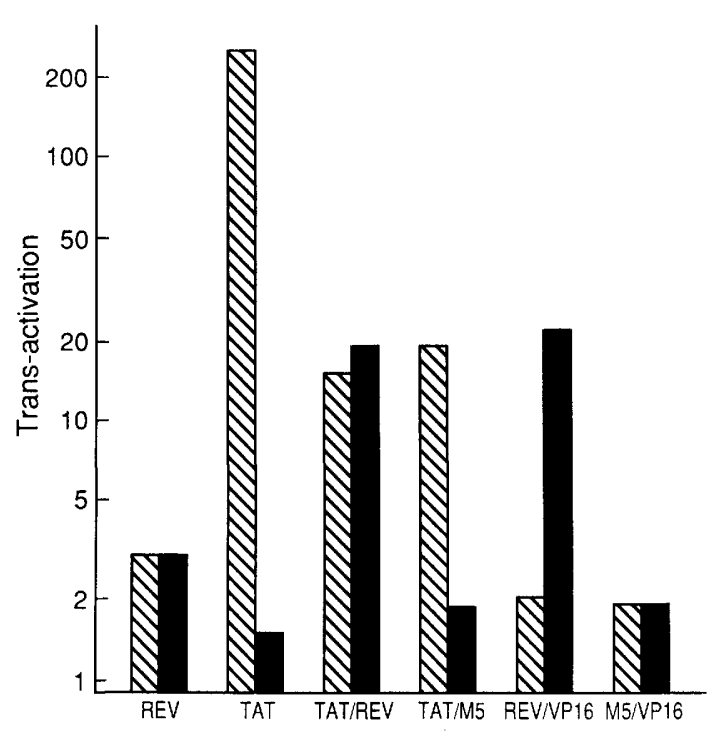

Figure 3. Trans-activation of the HIV-1 LTR in Jurkat T cells. Jurkat cells were transfected with either the pTAR/CAT (hatched bar) or the pSLIIB/CAT (solid bar) reporter plasmid, together with an equal amount of one of the indicated fusion protein expression plasmids. Trans-activation of the HIV-1 LTR by each of these proteins, as measured by CAT enzyme activity, is expressed as a multiple of the basal activity.
Table 4. RNA target sites for both Tat and VP16 must be promoter proximal

\begin{tabular}{lccc}
\hline & \multicolumn{3}{c}{ Relative trans-activation } \\
\cline { 2 - 4 } & + pcTAT & + pcTat/Rev & + pcRev/VP16 \\
\hline pTAT/CAT & 226 & 105 & - \\
pTAR64/CAT & 38 & 1.4 & - \\
pSLIIB/CAT & - & 123 & 27 \\
pSLIIB64/CAT & - & 2.8 & 1.3 \\
\hline
\end{tabular}

Assays were performed in HeLa cells as described in Table 1. These data represent the average of two separate transfection experiments. $(-)$ Not tested.

or SLIIB RNA targets, we constructed derivatives of pTAR/CAT and pSLIIB/CAT bearing a 64-bp insertion at a site located $8 \mathrm{bp}$ from the HIV-1 LTR cap site. In agreement with earlier reports (Selby et al. 1989), displacement of TAR 64 nucleotides away from the cap site, in pTAR64/CAT, was observed to inhibit trans-activation by Tat by $\sim 80 \%$ (Table 4). Surprisingly, trans-activation by the Tat/Rev fusion protein was almost completely abrogated by this small displacement of the RNA target site when assayed on either the TAR or SLIIB sequence. Similarly, Rev/VP16 also proved unable to activate the HIV-1 LTR when the SLIIB RNA target was moved away from the transcription start site.

\section{Rev/VP16 activates transcription from the HIV-1 LTR}

Trans-activation of the HIV-1 LTR by Tat occurs primarily at the transcriptional level (Hauber et al. 1987; Kao et al. 1987). We therefore used quantitative S1 nuclease analysis to assess the effect of the Tat/Rev and Rev/ VP16 proteins on the level of RNA expression directed by the HIV-1 LTR present in the pSLIIB/CAT plasmid (Fig. 4). The end-labeled probe used in this assay is able to quantitate CAT transcripts encoded by either pTAR/ CAT or pSLIIB/CAT and can also detect an internal control RNA, transcribed under the control of the Rous sarcoma virus (RSV) LTR present in the previously described pBC12 I plasmid (Berger et al. 1988), which does not encode CAT.

As predicted, the Tat/Rev fusion protein strongly activated the expression of the CAT mRNA encoded by the pTAR/CAT plasmid (Fig. 4, lanes 3,4). Similarly, both Tat/Rev and Rev/VP16, but not Tat or Rev alone, also strongly activated RNA expression from the pSLIIB/ CAT vector when compared with the nonresponsive internal control mRNA (Fig. 4, lanes 5-9). As observed previously with both Tat and other transcriptional transactivators (Cullen 1986; Wright et al. 1986; Laspia et al. 1990), the increase in the steady-state level of CAT mRNA does not appear sufficient to fully account for the increase in the level of CAT protein expression observed in the presence of the TAT/Rev and Rev/VP16 transactivators. It has been proposed (Laspia et al. 1990) that this may reflect the more efficient translational utilization of highly expressed mRNA species. In any event, 

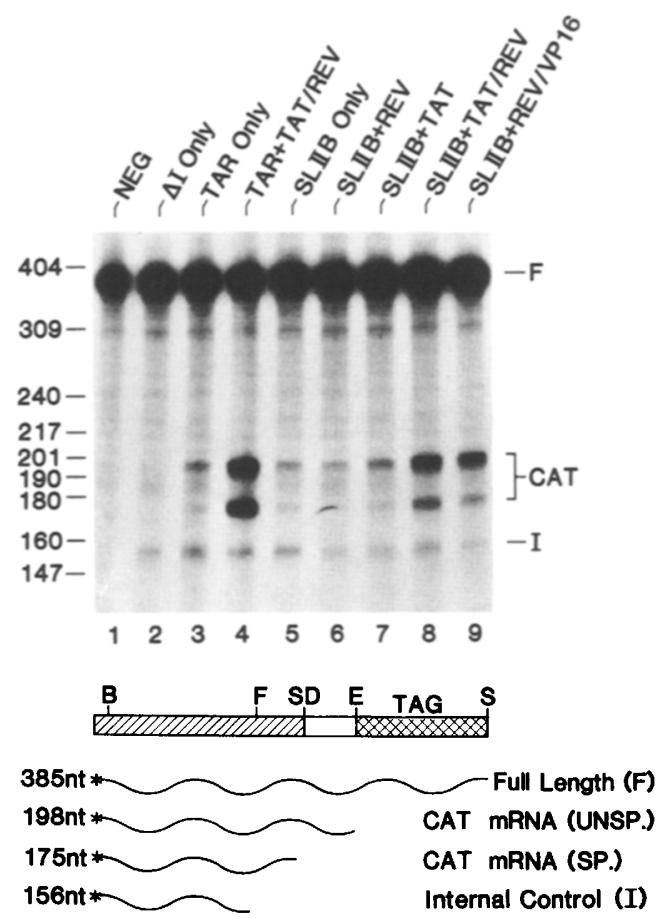

Figure 4. Quantitation of CAT RNA in transfected HeLa cells. HeLa cells were transfected with the indicator constructs pTAR/CAT (lanes 3,4) or pSLIIB/CAT (lanes 5-9), together with the indicated expression plasmids. At $48 \mathrm{hr}$ after transfection, total RNA was harvested and the levels of CAT-specific RNA were quantitated by SI nuclease analysis. The probe strategy used has been described (Berger et al. 1988). In brief, the full-length probe is end-labeled at a $B a m H I$ (B) site located at the $3^{\prime}$ end of the cat gene and extends through $3^{\prime}$ noncoding sequences, including an intron, derived from the rat preproinsulin gene. A pBR322-derived tag at the 3 ' end of the probe permits discrimination between the full-length probe $(F)$ and probe fragments rescued by either spliced (SP.) or unspliced (UNSP.) cat mRNA. Each culture was also cotransfected with the internal control plasmid $\mathrm{pBC} 12 \Delta \mathrm{I}$. The mRNA transcribed from the Tat nonresponsive RSV LTR present in this vector does not encode CAT but does rescue a 156-nucleotide probe fragment (I) truncated at the indicated FnuDII site (F). (E) EcoRI; (S) SstI; (SD) splice donor. (Lane 1) Culture transfected with pcTat/Rev only; (lane 2) culture transfected with pcTat/Rev $+\mathrm{pBC12 \Delta I}$.

these data do clearly demonstrate that the phenotypic effect of the interaction of Tat with TAR, when measured at either the protein or the RNA expression level, can be reproduced accurately by the interaction of Tat/ Rev or Rev/VP16 with the SLIIB RNA target.

\section{Discussion}

DNA sequence-specific transcription factors can frequently be dissected into two functional domains (Keegan et al. 1986; Ptashne 1988; Triezenberg et al. 1988; Mitchell and Tiian 1989). Sequence specificity is conferred by a binding domain that mediates the direct or indirect interaction of the transcription factor with the appropriate DNA target sites. A second domain, the tran- scriptional activation domain, permits the functional interaction of the factor with components of the cellular transcription machinery. The finding that these domains are entirely discrete in many transcriptional trans-activators has permitted the construction of functional chimeric proteins (Ptashne 1988; Southgate and Green 1991). This, in turn, has facilitated the identification of several protein motifs that mediate either sequence-specific DNA binding or transcriptional activation (Mitchell and Tjian 1989). Among the best characterized of the latter is the acidic activation domain first defined in the yeast protein GAL4 and found in a particularly potent form in the VP16 trans-activator of HSV (Sadowski et al. 1988).

The HIV-l Tat protein is unique in that this transcription factor has been shown to directly interact with an RNA, rather than a DNA, target sequence (Dingwall et al. 1990; Roy et al. 1990; Weeks et al. 1990). As in the case of DNA sequence-specific transcription factors, it has proven possible to construct functional chimeric proteins by fusing the activation domain of Tat to a heterologous sequence-specific RNA-binding protein (Selby and Peterlin 1990; Southgate et al. 1990). Here, we have used this approach to distinguish Rev sequences that are required for sequence-specific RNA binding in vivo from those required, more generally, for Rev function. However, the more important finding is that the activation domain contributed to the Tat/Rev fusion protein by Tat can be effectively substituted by the acidic transcriptional activation domain of VP16. The observation that these distinct transcriptional activation domains are functionally interchangeable has significant implications not only for the mechanism of action of Tat but also for transcriptional trans-activation in general.

\section{Implications for Rev function}

Analyses of the in vitro interaction of inactive Rev mutants with the RRE RNA target have suggested that these mutants can be divided into three distinct classes. Rev mutants, such as M10, that lack a functional copy of the leucine-rich activation motif appear indistinguishable from wild-type Rev in their ability to bind to, and multimerize on, the RRE (Olsen et al. 1990; Malim and Cullen 1991; Zapp et al. 1991). In contrast, mutants, such as M5, that lack a functional copy of the Rev arginine-rich motif are unable to bind to the RRE in vitro. The introduction of mutations either immediately amino- (M4) or carboxy-terminal (M7) to the argininerich motif results in inactive Rev proteins that have lost the ability to multimerize in vitro (Malim and Cullen 1991; Zapp et al. 1991). Although general agreement exists that multimerization is critical for Rev function, it has remained less clear whether Rev multimerization is an essential prerequisite for RRE binding (Olsen et al. 1990; Zapp et al. 1991) or is, instead, mediated by the interaction of Rev with the RRE substrate (Cook et al. 1991; Malim and Cullen 1991; Kjems et al. 1992; Tiley et al. 1992).

To identify Rev sequences required for specific RNA 
binding in vivo, we inserted the Rev mutations described above into the Tat/Rev or Rev/VP16 fusion protein context. As expected, introduction of the M5 mutation into the RNA-binding motif of Rev precluded the functional interaction of these fusion proteins with the SLIIB RNA target, although the Tat/M5 protein remained active on TAR (Tables 1 and 3). In contrast, mutation of the Rev activation motif, in Tat/M10, or inhibition of Rev multimerization, in M4/VP16 and M7/VP16, had little effect on trans-activation of the HIV-1 LTR via the SLIIB RNA target (Tables 1 and 3). Although these in vivo data cannot address the relative affinity of multimerization defective Rev proteins for the RRE, they do demonstrate that the arginine-rich domain is the only part of Rev that is essential for specific RNA binding in vivo (Böhnlein et al. 1991; Kjems et al. 1992).

Analysis of the RNA sequence specificity of Rev in vitro has suggested that the SLIIB subdomain of the RRE (Fig. 1) is both necessary and sufficient for Rev binding (Bartel et al. 1991; Heaphy et al. 1991; Tiley et al. 1992; Kjems et al. 1992). These in vitro data are confirmed by the highly efficient in vivo targeting of Tat/Rev and Rev/ VP16 to the HIV-1 LTR by the SLIIB RNA. We have also confirmed that the proposed 13-nucleotide core Revbinding site (Heaphy et al. 1991; Tiley et al. 1992) (Fig. 1) is not only critical for the in vivo interaction of Rev with SLIIB but also sufficient, albeit somewhat less effectively than the entire SLIIB RNA sequence (Table 2). The observation that the specific interaction of Rev with the RRE is mediated by identical small, highly discrete protein and nucleic acid sequences both in vitro and in vivo indicates that this binding event likely occurs independently of any cellular cofactor. In contrast, considerable evidence now suggests that the in vivo interaction of Tat with TAR is mediated by, and critically dependent on, a cellular RNA sequence-specific cofactor (Sullenger et al. 1991; Carroll et al. 1992).

\section{Implications for Tat function}

The functional interaction of Tat with TAR results in the transcriptional activation of the HIV-1 LTR (Hauber et al. 1987). Although Tat may enhance initiation to some extent (Laspia et al. 1990), it appears that the major effect of Tat both in vivo and in vitro is to induce efficient elongation by the otherwise poorly processive transcription complexes that initiate within the HIV-1 LTR (Kao et al. 1987; Marciniak et al. 1990; Feinberg et al. 1991; Kato et al. 1992). The apparent action of Tat as a processivity factor, and the fact that Tat acts via an RNA target, has appeared inconsistent with the hypothesis that Tat is mechanistically similar to eukaryotic transcription factors, such as VP16, that act via DNA target sites to primarily affect the level of transcription initiation (for review, see Cullen 1990).

In this paper we demonstrate that the acidic activation domain of VP16, when targeted to an RNA sequence by fusion to Rev, can activate gene expression (Tables 2 and 3) and transcription (Fig. 4) directed by the HIV-1 LTR promoter. The unexpected finding that the VP16 activa- tion domain can function effectively via a promoter proximal RNA target, when considered together with the recent observation that Tat is active when bound to upstream promoter DNA (Southgate and Green 1991), suggests that the mechanisms of action of these two trans-activators might well be similar. Consistent with this hypothesis, when targeted to either TAR or to a DNA sequence, Tat has been shown to cooperate effectively with the cellular transcription factor Spl but only poorly with acidic activators (Southgate and Green 1991; Kamine et al. 1991). Even more important is the observation of Southgate and Green (1991) that Tat is unable to activate further a TAR-containing HIV-1 LTR bearing multiple upstream GAL4-binding sites when tested in the presence of a high level of a GAL4-VP16 fusion protein. As noted by these investigators, the finding that the HIV-1 LTR can be maximally activated by this acidic activator appears inconsistent with the hypothesis that VP16 and Tat act at distinct steps in the transcription process, that is, initiation and elongation, respectively. Rather, this observation is more readily explained by the hypothesis that VP16 and Tat, whether bound to an RNA or a DNA target, are each acting on the same ratelimiting regulatory step.

\section{Implications for acidic activation domain function}

The mechanism of action of acidic activation domains remains unresolved (for review, see Roeder 1991). It has been suggested that VP16 might activate transcription by directly interacting with either the basal transcription factor TFIIB or, perhaps, with TFIID (Stringer et al. 1990; Lin and Green 1991). However, other evidence indicates that activation domains do not interact directly with the basal transcription machinery but, rather, with unidentified ancillary proteins that have been termed coactivators or adaptors (Berger et al. 1990; Hoffmann et al. 1990; Pugh and Tjian 1990; Dynlacht et al. 1991; Flanagan et al. 1991). Importantly, coactivator proteins appear dispensable for basal transcription but critical for activated transcription (Berger et al. 1990; Flanagan et al. 1991; Zhu and Prywes 1992). Nevertheless, it has generally been believed that acidic activators act by regulating the assembly of the preinitiation complex rather than by affecting, for example, the frequency of reinitiation from a preformed transcription initiation complex (Ptashne and Gann 1990; Lin and Green 1991; Roeder 1991).

In this paper we demonstrate that VP16 can efficiently activate transcription from the HIV-1 LTR when targeted to a nascent RNA sequence (Berkhout et al. 1989). Formation of this target RNA is dependent on transcription from the LTR, and a functional preinitiation complex must, therefore, have already been formed. As it is believed that initiation factors, including TFID, remain associated with the promoter during multiple rounds of initiation (Van Dyke et al. 1988; Katagiri et al. 1990), it seems probable that VP16, here, must be acting at a step subsequent to preinitiation complex assembly. The observation that VP16 function can be dissociated from any requirement for a stable interaction with a DNA target 
sequence appears inconsistent with scenarios for acidic activation domain function that require the formation on the DNA template of stable, multiprotein complexes involving VP16 and other cellular transcription factors (Ptashne and Gann 1990). Instead, it appears more likely that the RNA-bound VP16 activation domain serves to recruit cellular coactivator protein(s) to the vicinity of a preformed transcription initiation complex. These coactivators may then modify the HIV-1 LTR initiation complex so that the rate of transcription initiation is enhanced and/or, by analogy to Tat, render initiated transcription complexes resistant to premature termination.

Clearly, the observation that the activation domains of transcriptional trans-activators as apparently distinct as VP16 and Tat are functionally interchangeable falls well short of demonstrating that they act via the same mechanism. This finding, however, does strongly suggest that the mechanism of action of Tat is unlikely to be totally distinct from those utilized by VP16 and other conventional DNA sequence-specific trans-activators (Southgate and Green 1991). It therefore seems possible that transcriptional trans-activation of the HIV-1 LTR by Tat may provide a less specific target for chemotherapeutic intervention in HIV-1-infected individuals than has been adduced from its unique RNA target specificity (Hsu et al. 1991).

\section{Materials and methods \\ Construction of molecular clones}

The expression plasmids pcTat and pcRev contain cDNA copies of the HIV-1 Tat and Rev genes, respectively, under the control of the cytomegalovirus immediate early promoter present in the parental $\mathrm{pBC} 12 / \mathrm{CMV}$ plasmid. These constructs, and derivatives expressing mutant forms of Rev (pM4, pM5, pM7, pM10, pM14) or Tat (pK41A) have been described elsewhere (Malim et al. 1989a,b; Tiley et al. 1990). The pcTat/Rev expression plasmid, as well as mutated derivatives thereof, expresses fusion proteins consisting of the entire 86 -amino-acid Tat protein fused in-frame with the amino terminus of the 116-amino-acid Rev protein.

The expression plasmid pcRev/VP16 was derived by insertion of a BgIII-BamHI fragment, encoding the 78-amino-acid carboxy-terminal activation domain of VP16 (Triezenberg et al. 1988), into a BglII site located at amino acid position 113 in the fully active Rev missense mutant M14 (Malim et al. 1989a). Derivatives of pcRev/VP16 that express fusion proteins containing mutant forms of Rev (pM4/VP16, pM5/VP16, pM7/ VP16) or VP16 (pcRev/ $\triangle$ VP16) were obtained using standard recombinant DNA techniques. The pcRev/ $\Delta$ VP16 plasmid bears a missense mutation at VP16 amino acid 442 (Phe $\rightarrow$ Pro).

Previously, we have described an expression plasmid, $\mathrm{pBC12} /$ HIV/CAT, that contains the HIV-I LTR linked in cis to the indicator gene CAT (Berger et al. 1988). The $\mathrm{pTAR} / \mathrm{CAT}$ vector was derived from $\mathrm{pBCl} / \mathrm{HIV} / \mathrm{CAT}$ by insertion, between the HIV-1 LTR and the cat gene, of a 735-bp HindIII-EcoRV fragment derived from the $5^{\prime}$ nontranslated leader sequence of the Lansing strain of poliovirus type 2 . This region has been shown to permit cap-independent, internal initiation of translation (Pelletier and Sonenberg 1988) and was inserted to minimize any post-transcriptional effect of Tat on the expression of the CAT mRNAs (Hauber et al. 1987). Cleavage of pTAR/CAT at unique BglII (5'-AGATCT-3') and SacI (5'-GAGCTC-3') sites located within the DNA encoding the HIV-1 TAR element (Fig. 1) allowed the deletion of the 10-nucleotide apex of TAR. This short sequence was then replaced with a synthetic oligonucleotide (sense strand, 5'-GATCGAGCTGGGCGCAGCGCAAT GCGCTGACGGTAC-3') that introduced the 29-nucleotide SLIIB high-affinity Rev-binding site (Tiley et al. 1992), to give pSLIIB/CAT. This insert deletes the last pyrimidine residue of the TAR bulge but reintroduces the 4-bp stem located above the bulge (Fig. 1). An additional construct, termed pSLIIB-C/CAT, retained the proposed 13-nucleotide core SLIIB Rev-binding site (Heaphy et al. 1991; Tiley et al. 1992) but substituted a different, yet structurally similar, sequence for the 16-nucleotide apex of SLIIB. The converse construction, retaining the 16-nucleotide apical SLIIB sequence but bearing 7 substitution mutations within the 13-nucleotide core Rev-binding site (Fig. 1), was termed $\mathrm{p} \Delta \mathrm{SLIIB} / \mathrm{CAT}$.

To examine the effect of promoter proximity on the activity of the TAR or SLIIB RNA target, we first introduced a unique XhoI site 8 bp $3^{\prime}$ to the cap site of the HIV-1 LTR in both pTAR/CAT and pSLIIB/CAT, as described previously (Hauber and Cullen 1988). A 60-bp synthetic oligonucleotide (sense strand, 5'-TCGAACTCTAAGTGACTAGTTACAGTCGAAAACTATCAGTAAGTAGATCTTTGTTCCAAG-3') was then introduced at this site to give pTAR64/CAT and pSLIIB64/ CAT. This sequence was designed to lack significant secondary structure. Both orientations of the inserted sequence were observed to exert a similar inhibitory effect on trans-activation (Table 4 and data not shown). The pBC12 $\Delta$ I plasmid used as an internal control in the $\mathrm{Sl}$ nuclease analysis has been described (Berger et al. 1988). The pSV- $\beta$ gal construct was obtained from Promega (Madison, WI).

\section{Cell culture and transfection}

HeLa, Jurkat, and COS cells were maintained as described previously (Malim et al. 1991). HeLa cell cultures (35 mm) were transfected with $2 \mu \mathrm{g}$ of the CAT reporter construct and $2 \mu \mathrm{g}$ of the relevant expression plasmid using the calcium phosphate procedure (Cullen 1987). COS cell cultures (35 mm) were transfected with $125 \mathrm{ng}$ of the pgTAT indicator construct and $125 \mathrm{ng}$ of the relevant expression plasmid using DEAE-dextran and chloroquine (Cullen 1987). Jurkat cells were transfected as described by Grosschedl and Baltimore (1985). Assays for CAT and $\beta$-galactosidase activity were performed as described (Neumann et al. 1987; Rosenthal 1987) using HeLa and Jurkat cell lysates harvested at $\sim 48 \mathrm{hr}$ post-transfection.

\section{S1 nuclease protection analysis}

Total RNA was harvested from transfected HeLa cell cultures at $48 \mathrm{hr}$ post-transfection (Malim et al. 1989b). The 385-nucleotide probe utilized in this analysis was uniquely end-labeled at a BamHI site, located at the $3^{\prime}$ end of the CAT open reading frame, and spans $3^{\prime}$ noncoding sequences derived from the rat preproinsulin II gene that include part of an intron (Fig. 4) (Berger et al. 1988). A pBR322-derived 3' tag was attached at an intronic EcoRI site to facilitate discrimination between input probe and rescued probe fragments. As described in Figure 4, CAT mRNAs are predicted to rescue probe fragments of 198 or 175 nucleotides. A constant level of a previously described (Berger et al. 1988) internal control plasmid, termed pBC12 $\Delta \mathrm{I}$, was also cotransfected into each HeLa cell culture. The mRNA derived from $\mathrm{pBC} 12 \Delta \mathrm{I}$ does not encode CAT but does rescue a probe fragment of 156 nucleotides (Fig. 4). 


\section{Immunoprecipitation analysis}

Transfected COS cell cultures ( $35 \mathrm{~mm}$ ) were metabolically labeled at $48 \mathrm{hr}$ post-transfection using $\left[{ }^{35}\right.$ S]cysteine [Cullen 1987). The cultures were then lysed with $1 \mathrm{ml}$ of RIPA buffer, and equal portions of each cleared lysate were incubated with polyclonal rabbit antisera specific for either HIV-1 Tat or Rev (Malim et al. 1989b). The resultant immune complexes were precipitated with protein A-agarose, washed, resolved on $12 \%$ discontinuous SDS-polyacrylamide gels, and visualized by autoradiography.

\section{Acknowledgments}

We thank Pam Brown and Ted Benson for technical assistance and Sharon Goodwin for secretarial support. This work was supported by the Howard Hughes Medical Institute and U.S. Public Health Service grants AI28233, AI29821, and AI28662 from the National Institute of Allergy and Infectious Diseases.

The publication costs of this article were defrayed in part by payment of page charges. This article must therefore be hereby marked "advertisement" in accordance with 18 USC section 1734 solely to indicate this fact.

\section{References}

Bartel, D.P., M.L. Zapp, M.R. Green, and J.W. Szostak. 1991. HIV-1 Rev regulation involves recognition of non-WatsonCrick base pairs in viral RNA. Cell 67: 529-536.

Berger, J., J. Hauber, R. Hauber, R. Geiger, and B.R. Cullen. 1988. Secreted placental alkaline phosphatase: A powerful new quantitative indicator of gene expression in eukaryotic cells. Gene 66: 1-10.

Berger, S.L., W.D. Cress, A. Cress, S.J. Triezenberg, and L. Guarente. 1990. Selective inhibition of activated but not basal transcription by the acidic activation domain of VP16: Evidence for transcriptional adaptors. Cell 61: 1199-1208.

Berkhout, B. and K.-T. Jeang. 1989. Trans-activation of human immunodeficiency virus type $l$ is sequence specific for both the single-stranded bulge and loop of the trans-acting-responsive hairpin: A quantitative analysis. J. Virol. 63: 55015504.

Berkhout, B., R.H. Silverman, and K.-T. Jeang. 1989. Tat transactivates the human immunodeficiency virus through a nascent RNA target. Cell 59: 273-282.

Böhnlein, E., J. Berger, and J. Hauber. 1991. Functional mapping of the human immunodeficiency virus type 1 Rev RNA binding domain: New insights into the domain structure of Rev and Rex. J. Virol. 65: 7051-7055.

Bradford, M.M. 1976. A rapid and sensitive method for the quantitation of microgram quantities of protein utilizing the principle of protein-dye binding. Anal. Biochem. 72: 248254.

Carroll, R., L. Martarano, and D. Derse. 1991. Identification of lentivirus Tat functional domains through generation of equine infectious anemia virus/human immunodeficiency virus type 1 tat gene chimeras. I. Virol. 65: 3460-3467.

Carroll, R., B.M. Peterlin, and D. Derse. 1992. Inhibition of human immunodeficiency virus type 1 Tat activity by coexpression of heterologous trans activators. J. Virol. 66: 20002007.

Cook, K.S., G.J. Fisk, J. Hauber, N. Usman, T.J. Daly, and J.R. Rusche. 1991. Characterization of HIV-1 REV protein: Binding stoichiometry and minimal RNA substrate. Nucleic Acids Res. 19: 1577-1583.
Cress, W.D. and S.J. Triezenberg. 1991. Critical structural elements of the VP16 transcriptional activation domain. Science 251: 87-90.

Cullen, B.R. 1986. Trans-activation of human immunodeficiency virus occurs via a bimodal mechanism. Cell 46: 973-982. . 1987. Use of eukaryotic expression technology in the functional analysis of cloned genes. Methods Enzymol. 152: 684-704.

1990. The HIV-1 Tat protein: An RNA sequence-specific processivity factor? Cell 63: 655-657.

Daly, T.J., K.S. Cook, G.S. Gray, T.E. Maione, and J.R. Rusche. 1989. Specific binding of HIV-1 recombinant Rev protein to the Rev-responsive element in vitro. Nature 342: 816-819.

Dayton, A.I., J.G. Sodroski, C.A. Rosen, W.C. Goh, and W.A. Haseltine. 1986. The trans-activator gene of the human $T$ cell lymphotropic virus type III is required for replication. Cell 44: 941-947.

Dingwall, C., I. Ernberg, M.J. Gait, S.M. Green, S. Heaphy, J. Karn, A.D. Lower, M. Singh, and M.A. Skinner. 1990. HIV-1 tat protein stimulates transcription by binding to a U-rich bulge in the stem of the TAR RNA structure. EMBO $J$. 9: 4145-4153.

Dynlacht, B.D., T. Hoey, and R. Tjian. 1991. Isolation of coactivators associated with the TATA-binding protein that mediate transcriptional activation. Cell 66: 563-576.

Emerman, M., R. Vazeux, and K. Peden. 1989. The rev gene product of the human immunodeficiency virus affects envelope-specific RNA localization. Cell 57: 1155-1165.

Feinberg, M.B., D. Baltimore, and A.D. Frankel. 1991. The role of Tat in the human immunodeficiency virus life cycle indicates a primary effect on transcriptional elongation. Proc. Natl. Acad. Sci. 88: 4045-4049.

Feng, S. and E.C. Holland. 1988. HIV-1 tat trans-activation requires the loop sequence within tar. Nature 334: 165-167.

Fisher, A.G., M.B. Feinberg, S.F. Josephs, M.E. Harper, L.M. Marselle, G. Reyes, M.A. Gonda, A. Aldovini, C. Debouk, R.C. Gallo, and F. Wong-Staal. 1986. The trans-activator gene of HTLV-III is essential for virus replication. Nature 320: $367-371$.

Flanagan, P.M., R.J. Kelleher III, M.H. Sayre, H. Tschochner, and R.D. Kornberg. 1991. A mediator required for activation of RNA polymerase II transcription in vitro. Nature 350: 436438.

Green, M., M. Ishino, and P.M. Loewenstein. 1989. Mutational analysis of HIV-1 Tat minimal domain peptides: Identification of trans-dominant mutants that suppress HIV-LTRdriven gene expression. Cell 58: 215-223.

Grosschedl, R., and D. Baltimore. 1985. DEAE-dextran transfection of lymphoid cell lines. Cell 41: 885.

Hammarskjöld, M.-L., J. Heimer, B. Hammarskjöld, I. Sangwan, L. Albert, and D. Rekosh. 1989. Regulation of human immunodeficiency virus env expression by the rev gene product. $J$. Virol. 63: 1959-1966.

Hauber, J. and B.R. Cullen. 1988. Mutational analysis of the trans-activation-responsive region of the human immunodeficiency virus type 1 long terminal repeat. $/$. Virol. 62: 673679.

Hauber, J., A. Perkins, E.P. Heimer, and B.R. Cullen. 1987. Trans-activation of human immunodeficiency virus gene expression is mediated by nuclear events. Proc. Natl. Acad. Sci. 84: 6364-6368.

Heaphy, S., J.T. Finch, M.J. Gait, J. Karn, and M. Singh. 1991. Human immunodeficiency virus type 1 regulator of virion expression, rev, forms nucleoprotein filaments after binding to a purine-rich "bubble" located within the rev-responsive region of viral mRNAs. Proc. Natl. Acad. Sci. 88: 7366- 
7370.

Hoffmann, A., E. Sinn, T. Yamamoto, J. Wang, A. Roy, M. Horikoshi, and R.G. Roeder. 1990. Highly conserved core domain and unique $\mathrm{N}$ terminus with presumptive regulatory motifs in a human TATA factor (TFIID). Nature 346: 387-390.

Hsu, M.-C., A.D. Schutt, M. Holly, L.W. Slice, M.I. Sherman, D.D. Richman, M.J. Potash, and D.J. Volsky. 1991. Inhibition of HIV replication in acute and chronic infections in vitro by a Tat antagonist. Science 254: 1799-1802.

Jakobovits, A., D.H. Smith, E.B. Jakobovits, and D.J. Capon. 1988. A discrete element $3^{\prime}$ of human immunodeficiency virus 1 (HIV-1) and HIV-2 mRNA initiation sites mediates transcriptional activation by an HIV trans-activator. Mol. Cell. Biol. 8: 2555-2561.

Kamine, J., T. Subramanian, and G. Chinnadurai. 1991. Sp1dependent activation of a synthetic promoter by human immunodeficiency virus type 1 Tat protein. Proc. Natl. Acad. Sci. 88: 8510-8514.

Kao, S.-Y., A.F. Calman, P.A. Luciw, and B.M. Peterlin. 1987. Anti-termination of transcription within the long terminal repeat of HIV-1 by tat gene product. Nature 330: 489-493.

Katagiri, F., K.-I. Yamazaki, M. Horikoshi, R.G. Roeder, and N.-H. Chua. 1990. A plant DNA-binding protein increases the number of active preinitiation complexes in a human in vitro transcription system. Genes \& Dev. 4: 1899-1909.

Kato, H., H. Sumimoto, P. Pognonec, C.-H. Chen, C.A. Rosen, and R.G. Roeder. 1992. HIV-1 Tat acts as a processivity factor in vitro in conjunction with cellular elongation factors. Genes \& Dev. 6: 655-666.

Keegan, L., G. Gill, and M. Ptashne. 1986. Separation of DNA binding from the transcription-activating function of a eukaryotic regulatory protein. Science 231: 699-704.

Kjems, J., B.J. Calnan, A.D. Frankel, and P.A. Sharp. 1992. Specific binding of a basic peptide from HIV-1 Rev. EMBO I. 11: 1119-1129.

Laspia, M.F., A.P. Rice, and M.B. Mathews. 1990. Synergy between HIV-1 Tat and adenovirus ElA is principally due to stabilization of transcriptional elongation. Genes \& Dev. 4: 2397-2408.

Lazinski, D., E. Grzadzielska, and A. Das. 1989. Sequence-specific recognition of RNA hairpins by bacteriophage antiterminators requires a conserved arginine-rich motif. Cell 59: 207-218.

Lin, Y.-S. and M.R. Green. 1991. Mechanism of action of an acidic transcriptional activator in vitro. Cell 64: 971-981.

Malim, M.H. and B.R. Cullen. 1991. HIV-1 structural gene expression requires the binding of multiple Rev monomers to the viral RRE: Implications for HIV-1 latency. Cell 65: 241248.

Malim, M.H., S. Böhnlein, J. Hauber, and B.R. Cullen. 1989a. Functional dissection of the HIV-l Rev trans-activatorDerivation of a trans-dominant repressor of Rev function. Cell 58: 205-214.

Malim, M.H., J. Hauber, S.-Y. Le, J.V. Maizel, and B.R. Cullen. 1989b. The HIV-1 rev trans-activator acts through a structured target sequence to activate nuclear export of unspliced viral mRNA. Nature 338: 254-257.

Malim, M.H., L.S. Tiley, D.F. McCarn, J.R. Rusche, J. Hauber, and B.R. Cullen. 1990. HIV-1 structural gene expression requires binding of the Rev trans-activator to its RNA target sequence. Cell 60: 675-683.

Marciniak, R.A., B.J. Calnan, A.D. Frankel, and P.A. Sharp. 1990. HIV-1 Tat protein trans-activates transcription in vitro. Cell 63: 791-802.

Mitchell, P.J. and R. Tjian. 1989. Transcriptional regulation in mammalian cells by sequence-specific DNA binding pro- teins. Science 245: 371-378.

Neumann, J.R., C.A. Morency, and K.O. Russian. 1987. A novel rapid assay for chloramphenicol acetyltransferase gene expression. BioTechniques 5: 444-447.

Olsen, H.S., A.W. Cochrane, P.J. Dillon, C.M. Nalin, and C.A. Rosen. 1990. Interaction of the human immunodeficiency virus type $1 \mathrm{Rev}$ protein with a structured region in env mRNA is dependent on multimer formation mediated through a basic stretch of amino acids. Genes \& Dev. 4: $1357-1364$.

Pelletier, J. and N. Sonenberg. 1988. Internal initiation of translation of eukaryotic mRNA directed by a sequence derived from poliovirus RNA. Nature 334: 320-325.

Ptashne, M. 1988. How eukaryotic transcriptional activators work. Nature 335: 683-689.

Ptashne, M. and A.A.F. Gann. 1990. Activators and targets. Nature 346: 329-331.

Pugh, B.F. and R. Tiian. 1990. Mechanism of transcriptional activation by Spl: Evidence for coactivators. Cell 61: 11871197.

Roeder, R.G. 1991. The complexities of eukaryotic transcription initiation: Regulation of preinitiation complex assembly. Trends Biochem. Sci. 16: 402-441.

Rosen, C.A., E. Terwilliger, A. Dayton, J.G. Sodroski, and W.A. Haseltine. 1988. Intragenic cis-acting art gene-responsive sequences of the human immunodeficiency virus. Proc. Natl. Acad. Sci. 85: 2071-2075.

Rosenthal, N. 1987. Identification of regulatory elements of cloned genes with functional assays. Methods Enzymol. 152: $704-720$.

Roy, S., U. Delling, C.-H. Chen, C.A. Rosen, and N. Sonenberg. 1990. A bulge structure in HIV-1 TAR RNA is required for Tat binding and Tat-mediated trans-activation. Genes \& Dev. 4: 1365-1373.

Sadowski, I., J. Ma, S. Triezenberg, and M. Ptashne. 1988. GAL4VP16 is an unusually potent transcriptional activator. $\mathrm{Na}$ ture 335: 563-564.

Selby, M. and B.M. Peterlin. 1990. Trans-activation by HIV-1 Tat via a heterologous RNA binding protein. Cell 62: 769 776.

Selby, M.J., E.S. Bain, P.A. Luciw, and B.M. Peterlin. 1989. Structure, sequence, and position of the stem-loop in tar determine transcriptional elongation by tat through the HIV-1 long terminal repeat. Genes \& Dev. 3: 547-558.

Sodroski, J., W.C. Goh, C. Rosen, A. Dayton, E. Terwilliger, and W. Haseltine. 1986. A second post-transcriptional trans-activator gene required for HTLV-III replication. Nature 321: 412-417.

Southgate, C.D. and M.R. Green. 1991. The HIV-1 Tat protein activates transcription from an upstream DNA-binding site: Implications for Tat function. Genes \& Dev. 5: 2496-2507.

Southgate, C., M.L. Zapp, and M.R. Green. 1990. Activation of transcription by HIV-1 Tat protein tethered to nascent RNA through another protein. Nature 345: 640-642.

Stringer, K.F., C.J. Ingles, and J. Greenblatt. 1990. Direct and selective binding of an acidic transcriptional activation domain to the TATA-box factor TFIID. Nature 345: 783-786.

Sullenger, B.A., H.F. Gallardo, G.E. Ungers, and E. Gilboa. 1991. Analysis of trans-acting response decoy RNA-mediated inhibition of human immunodeficiency virus type 1 transactivation. J. Virol. 65: 6811-6816.

Tiley, L.S., P.H. Brown, and B.R. Cullen. 1990. Does the human immunodeficiency virus Tat trans-activator contain a discrete activation domain? Virology 178: 560-567.

Tiley, L.S., M.H. Malim, H.K. Tewary, P.G. Stockley, and B.R. Cullen. 1992. Identification of a high-affinity RNA-binding 
site for the human immunodeficiency virus type 1 Rev protein. Proc. Natl. Acad. Sci. 89: 758-762.

Triezenberg, S.J., R.C. Kingsbury, and S.L. McKnight. 1988. Functional dissection of VP16, the trans-activator of herpes simplex virus immediate early gene expression. Genes \& Dev. 2: 718-729.

Van Dyke, M.W., R.G. Roeder, and M. Sawadogo. 1988. Physical analysis of transcription preinitiation complex assembly on a class II gene promoter. Science 241: 1335-1338.

Weeks, K.M., C. Ampe, S.C. Schultz, T.A. Steitz, and D.M. Crothers. 1990. Fragments of the HIV-1 Tat protein specifically bind TAR RNA. Science 249: 1281-1285.

Wright, C.M., B.K. Felber, H. Paskalis, and G.N. Pavlakis. 1986. Expression and characterization of the trans-activator of HTLV-III/LAV virus. Science 234: 988-992.

Zapp, M.L. and M.R. Green. 1989. Sequence-specific RNA binding by the HIV-1 Rev protein. Nature 342: 714-716.

Zapp, M.L., T.J. Hope, T.G. Parslow, and M.R. Green. 1991. Oligomerization and RNA binding domains of the type 1 human immunodeficiency virus Rev protein: A dual function for an arginine-rich binding motif. Proc. Natl. Acad. Sci. 88: 7734-7738.

Zhu, H. and R. Prywes. 1992. Identification of a coactivator that increases activation of transcription by serum response factor and GAL4-VP16 in vitro. Proc. Natl. Acad. Sci. 89: 52915295. 


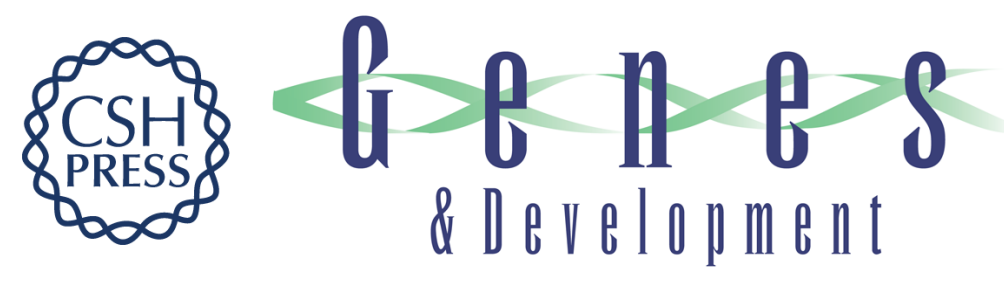

\title{
The VP16 transcription activation domain is functional when targeted to a promoter-proximal RNA sequence.
}

\author{
L S Tiley, S J Madore, M H Malim, et al.
}

Genes Dev. 1992, 6:

Access the most recent version at doi:10.1101/gad.6.11.2077

$\begin{array}{ll}\text { References } & \begin{array}{l}\text { This article cites } 74 \text { articles, } 30 \text { of which can be accessed free at: } \\ \text { http://genesdev.cshlp.org/content/6/11/2077.full.html\#ref-list-1 }\end{array}\end{array}$

License

Email Alerting Receive free email alerts when new articles cite this article - sign up in the box at the top Service right corner of the article or click here.

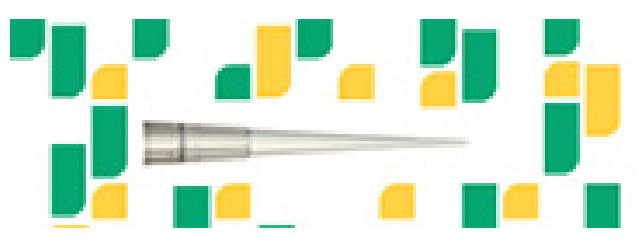

Focused on your science. 